\title{
The natural history of conjunctival naevi in children and adolescents
}

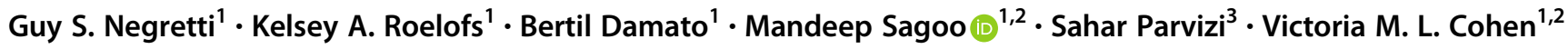

Received: 25 August 2020 / Revised: 28 October 2020 / Accepted: 29 October 2020 / Published online: 16 November 2020

(c) The Author(s), under exclusive licence to The Royal College of Ophthalmologists 2020

\begin{abstract}
Objective The objective of this study is to characterise the natural history of conjunctival naevi in a paediatric and adolescent population.

Methods All children and adolescents referred to Moorfields Ocular Oncology Service for evaluation between January 2015 and 2020 were included. Exclusion criteria included age $>20$ years old and lack of anterior segment photographs. A total of 77 patients were included with a mean age of 12 years (standard deviation: 3.9; range, 4-20). The main outcome measures were: number of conjunctival naevi that grew, changed in pigmentation, required excisional biopsy, or were histologically malignant. If there was growth, the percentage increase in size was measured.

Results At their first visit, $13 \%$ of patients (10/77) were discharged to local follow-up and $10 \%$ (8/77) proceeded to excisional biopsy, four further patients underwent excisional biopsy after a period of follow-up. On histopathological assessment, 92\% (11/12) of lesions were benign conjunctival naevi. One patient, who had suspicious clinical features at presentation, had conjunctival melanoma. Fifty-nine patients were followed over a median of 1.1 years (interquartile range: 1.54; range, 3 months to 4 years). Eight per cent (5/59) of conjunctival naevi enlarged in diameter by a mean percentage increase in size of 2\%, whereas 5\% (3/59) showed increased pigmentation and 8.5\% (5/59) showed decreased pigmentation. Conclusions Growth of conjunctival naevi in children is infrequent $(8 \%)$ and the large majority of those excised are benign. Because of a lack of evidence, these patients are often followed for years in ophthalmic practice. This series demonstrates that prolonged follow-up may not be necessary.
\end{abstract}

\section{Introduction}

Conjunctival naevi account for $61 \%$ of tumours in children in a recent case series of tumours referred to a large ocular oncology service in the USA [1]. Clinically, compared to naevi, conjunctival melanomas are thicker, with larger basal diameters, lacking cysts and having prominent feeder vessels and intrinsic vasculature [1]. Pigmented lesions involving the cornea or located in palpebral conjunctiva, plica or caruncle also raise suspicion for conjunctival melanoma [2].

$\triangle$ Guy S. Negretti

g.negretti@nhs.net

1 Department of Ocular Oncology, Moorfields Eye Hospital, City Road, London EC1V 2PD, UK

2 NIHR Biomedical Research Centre for Ophthalmology at Moorfields Eye Hospital and University College London Institute of Ophthalmology, London, UK

3 Department of Ophthalmology, Surrey and Sussex Healthcare NHS Trust, Canada Ave, Redhill RH1 5RH, UK
While there have been two previously published large case series reporting the natural history of conjunctival naevi, the mean age at presentation in both of these studies was $>30$ years $[2,3]$. Both these studies demonstrated similar clinical findings. For example, most naevi were located in bulbar locations in horizontal meridians, either temporally or nasally. Both studies found growth of naevi in some patients (8 and 4\%). This growth was, in general, not associated with malignancy, which was rare $(0.7$ and $0 \%)$ $[2,3]$. Our impression, in clinical practice, not previously published, is that most conjunctival naevi appear and enlarge in the second half of the first decade of life.

To the best of our knowledge, there are no sizeable case series in the literature reporting the natural history of conjunctival naevi in children and adolescents. Because of this, a cautious approach is often adopted so that these patients tend to be followed for several years. As families often live far from the hospital, ongoing regular visits can be costly and disruptive to schooling. The purpose of this study was to describe the natural history of conjunctival naevi in children and adolescents, with the aim of improving 
evidence-based management with respect to biopsy, followup protocols and family counselling.

\section{Methods}

This is a single centre retrospective case series study. The electronic patient record was searched for the key term 'conjunctival naevus' to identify patients referred to the Moorfields Eye Hospital Ocular Oncology Service for evaluation between January 2015 and January 2020. Exclusion criteria included age $>20$ years at first visit even if the naevus had been noticed prior to their 20th birthday, and insufficient photographic documentation of the naevi to allow analysis. This meant that at least two sequential photographs were required for all subjects who underwent a period of observation rather than excision at their first visit. There was no specific minimum follow-up time.

Clinical notes were reviewed for demographic data, including age, sex and ethnicity, referring clinician details (i.e., optician, general practitioner and ophthalmologist), tumour laterality and past medical history. Lesion characteristics recorded included: iris colour; lesion location, size and colour; and the presence or absence of cysts, feeder vessels, intrinsic vessels and hair. For patients who were examined more than once, sequential colour photographs were examined by the authors (GSN and KAR) for (a) change in lesion size and/or (b) change in pigmentation. Where a change in size was noted, the change in area of the naevus was measured using the SketchAndCalc ${ }^{\mathrm{TM}}$ application to define the contour of the naevus to calculate the area of the lesion (see Fig. 1). If, on a particular visit, no photo was taken then we relied on the clinical notes to inform us of any change.

Anterior segment OCT was not routinely performed, so changes in thickness were estimated from the photographs.

Descriptive statistics were used to estimate mean \pm standard deviation (SD) (range) when normally distributed, and median (interquartile range (IQR), range) when not. This study was approved by the Institutional Review Board at Moorfields Eye Hospital (CA20/ONC/607). The study adhered to the tenets of the Declaration of Helsinki.

\section{Results}

Between January 2015 and January 2020, 92 children/ adolescents were referred to Moorfields Eye Hospital Ocular Oncology Service for evaluation of conjunctival naevi. Fifteen patients were excluded because of poor photographic documentation of the naevus, leaving a total of 77 cases. There were more males $(47 / 77 ; 61 \%)$ than females $(30 / 77 ; 39 \%)$ and left and right eyes were affected in approximately equal numbers $(36 / 77 ; 47 \%$ and $41 / 77$; $53 \%$, respectively). The mean age at presentation was 12 years (SD: 3.9; range, 4-20). Approximately one-third of patients were white $(24 / 77 ; 31 \%)$ (Table 1$)$.

Table 2 summarises the ocular and naevus findings of all patients at first presentation and those who were followed up at MEH. Most naevi were seen in brown-eyed individuals $(60 / 77 ; 78 \%)$. Most $(74 / 77 ; 96 \%)$ naevi were located in the bulbar conjunctiva. They tended to be either in the temporal $(41 / 77 ; 53 \%)$ or nasal $(32 / 77 ; 42 \%)$ quadrants. No naevi were found in the tarsal, forniceal or inferior bulbar conjunctiva. Cysts were frequently observed $(63 / 77 ; 82 \%)$. Most naevi were brown $(44 / 77 ; 57 \%)$. Feeder vessels were observed in 21/77 (27\%) of patients. Intrinsic vessels were present in $24 / 77(31 \%)$. Hair was observed in one caruncular naevus. In $57 \%$ of cases (44/77), the posterior border of the naevus involved the limbus, and in one case $(1 / 77 ; 1.3 \%)$ the naevus involved the cornea.

At their first visit, eight children were listed for excisional biopsy: seven because of patient request and one because of features suspicious of malignancy at presentation (Fig. 2).

All the other children underwent a period of observation, 59 under the Ocular Oncology Service at Moorfields and 10 with their local general ophthalmologist. Data from the local ophthalmologists were not attained so these children were effectively lost to follow-up from this study. All the 59 children monitored at Moorfields maintained follow-up until they were discharged from the clinic or the study finished. Median follow-up for children at Moorfields was 1.1 years (IQR: 1.54 ; range, 3 months to 4 years). A summary of the patients' management is shown in Table 1.

During this follow-up period at Moorfields (mean: 1.1 years), $5 / 59 ; 8.5 \%$ of naevi showed an increase in diameter. The average percentage increase in area was $20 \%$ (range, $2-60)$. 2/59; $3 \%$ of these naevi also had an associated increase in pigmentation and, conversely, 2/59; 3 showed a decrease in pigmentation. $3 / 59$; another $5 \%$ children had no growth but increased pigmentation and 5/59; and $8.5 \%$ had no growth but decreased pigmentation. No naevi had an increase in thickness. In our series, conjunctival naevi tended to depigment in older children whereas documented growth tended to occur in younger children; however, these differences were not statistically significant (two sample $t$-tests, $p=0.9$ and $p=0.23$ ). Changes in pigment or size were not seen in association with topical drops (e.g., antihistamine or steroid).

We found progression, whether enlargement or change in colour, in 13/59 (22\%) cases; however, during the followup period, only four children underwent excisional biopsy: Two due to patient request and two because of increased pigment or growth. Therefore, most of the documented change was only monitored clinically. All biopsies were 

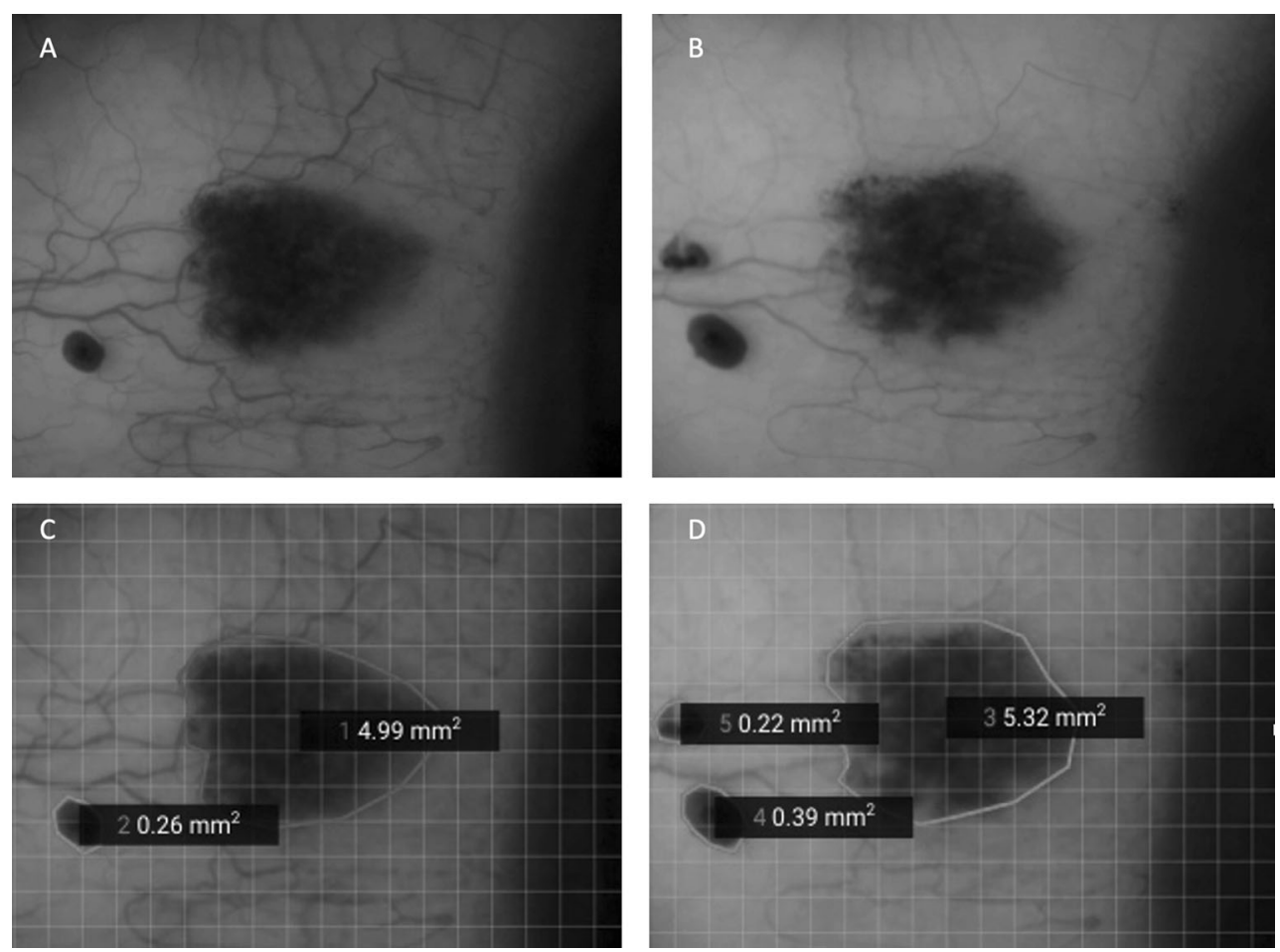

Fig. 1 Example of a conjunctival naevus that grew. At presentation (A) and 5 months later (B). The SketchAndCalc Application was used to measure the area before $(\mathbf{C})$ and after $(\mathbf{D})$ growth.

performed using the 'no touch' technique. The histopathology of the 12 biopsied tumours revealed: 8 compound naevi, 1 junctional naevus, 1 combined naevus, 1 intraepithelial naevus and 1 melanoma.

The one case of conjunctival melanoma occurred in a 18year old, white male (Fig. 2). As there was a high preoperative suspicion of melanoma, double freeze thaw cryotherapy was applied at the time of surgery and the conjunctival defect was closed using an amniotic membrane graft. The diagnosis of melanoma was confirmed using four-colour FISH. The tumour thickness was $1 \mathrm{~mm}$. Because the tumour involved the lateral and deep margins histologically but not clinically, this patient was treated with adjunctive strontium brachytherapy post biopsy and has been followed for 5 years with no evidence of tumour recurrence. The naevus that was biopsied because of growth was a compound naevus with some nuclear pleomorphism in the junctional component so the diagnosis of naevus was confirmed with four-colour FISH. The naevus biopsied due to increased pigmentation was a junctional naevus.

\section{Discussion}

There are many similarities between our results and those looking at conjunctival naevi predominantly in adults (Table 3) [2, 3].
Like the other studies, most naevi in our study were located on the bulbar conjunctiva (95\%), were either in the temporal $(53 \%)$ or nasal $(42 \%)$ horizontal quadrants, with cysts (82\%) and often involving the limbus (57\%). All these features have been recognised previously as being more commonly seen in naevi than melanomas and thus are signs clinicians specifically look for when assessing likelihood of malignancy $[2,4]$. The fact that most naevi in this study had these reassuring clinical signs is reflected in the benign histopathology and the lack of malignant transformation of the naevi. The one naevus that had worrisome clinical features at presentation $(9 \mathrm{~mm}$ largest basal diameter, corneal involvement and recurrence at the site of a previously excised atypical naevus) proved to be histologically malignant.

It is interesting that in our study $31 \%$ of patients were white, whilst $34 \%$ were black or Asian. This compares to $89 \%$ white in the study by Shields et al. [2] and $85 \%$ in the study by Levecq et al. [3]. This reflects the multicultural population of London and the peri-London location of our referrers especially for paediatric and adolescent patients.

The histopathology from the excised naevi demonstrated a predominance of compound naevi, which is similar to previous studies $[2,3,5,6]$. We did not note a significant percentage of junctional naevi in our study; this differs from previous reports, which have noted significant junctional activity in conjunctival naevi in children, as compared to 
Table 1 Patient demographics and a summary of the management of the naevi in this study.

\begin{tabular}{ll}
\hline & Number $(\%)$ \\
\hline Demographics & \\
Age at presentation mean \pm SD, (range), years & $12 \pm 3.9,(4-19)$ \\
Male & $47(61 \%)$ \\
Female & $30(39 \%)$ \\
Race & \\
White & $24(31 \%)$ \\
Black & $10(13 \%)$ \\
Asian & $16(21 \%)$ \\
Mixed & $1(1 \%)$ \\
Unknown & $22(29 \%)$ \\
Other & $4(5 \%)$ \\
Underlying systemic condition & $1(1.3 \%)$ \\
Wilms' tumour & $1(1.3 \%)$ \\
Eczema & $1(1.3 \%)$ \\
Anal stenosis/solitary kidney & $1(1.3 \%)$ \\
Asperger's syndrome & $1(1.3 \%)$ \\
Ex premature (35 weeks) & $1(8.3 \%)$ \\
Management & $1(8.3 \%)$ \\
Observation & $1(8.3 \%)$ \\
Excisional biopsy & $1(84 \%)$ \\
Reason for excision & $1(8 \%)$ \\
Patient request & \\
Suspicion of melanoma at presentation & $1(75 \%)$ \\
Growth during observation period & $1(8.3 \%)$ \\
Increased pigment during observation period & $1(8.6 \%)$ \\
Comtraepithelial nevus & \\
Junctional nevus & \\
Combined nevus & \\
\hline
\end{tabular}

adults $[2,5,6]$. It is difficult to infer too much from this finding given the small numbers of naevi excised in this study. In common with results previously reported by Levecq et al., the major reason for excising lesions in this study was patient request: $75 \%$ in this study and $83 \%$ in Levecq et al. [3]. This is in contrast to Shields et al. [2], who reported that only $10 \%$ of lesions were excised for cosmetic reasons (see Table 3). Patient or parent request was the main indication for surgery in our cohort; this is not surprising, especially as the mean age of the patients was 12 years and therefore the parents were involved in the consent process. In our experience, the opinions of the family especially related to cancer phobias are often greater than the concerns of the patient in this younger age group. As children become teenagers, the request for surgical excision is more
Table 2 Summary of ocular and naevus findings of all naevi at presentation and of those 59 cases followed up at MEH.

\begin{tabular}{lll}
\hline Ocular/nevus findings & All nevi $(\%)$ & $\begin{array}{l}\text { Monitored } \\
\text { nevi }(\%)\end{array}$ \\
\hline $\begin{array}{ll}\text { Left eye } \\
\text { Right eye }\end{array}$ & $36(47 \%)$ & $30(51 \%)$ \\
Iris colour & $41(53 \%)$ & $29(49 \%)$ \\
$\quad$ Blue & & \\
$\quad$ Green & $9(12 \%)$ & $8(14 \%)$ \\
$\quad$ Brown & $6(8 \%)$ & $4(7 \%)$ \\
$\quad$ Unknown & $60(78 \%)$ & $45(76 \%)$ \\
Naevus location & $2(2 \%)$ & $2(3 \%)$ \\
$\quad$ Bulbar & & \\
$\quad$ Caruncle & $74(96 \%)$ & $56(95 \%)$ \\
Quadrant & $3(4 \%)$ & $3(5 \%)$ \\
$\quad$ Temporal & & \\
$\quad$ Nasal & $41(53 \%)$ & $30(51 \%)$ \\
$\quad$ Superior & $32(42 \%)$ & $26(44 \%)$ \\
$\quad$ Inferior & $4(5 \%)$ & $3(5 \%)$ \\
Largest basal diameter, mean \\
(range), mm
\end{tabular}

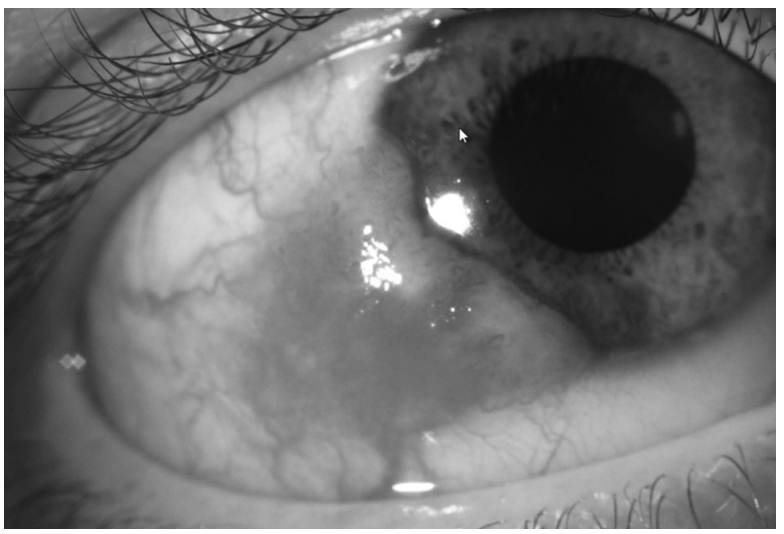

Fig. 2 Photograph of the naevus that presented with features suspicous of malignancy. These features were: largest basal diameter $9 \mathrm{~mm}$, corneal involvement, feeder vessels and recurrence at the site of a previously excised atypical naevus.

often based on cosmetic concerns, which may arise out of peer pressure. 
Table 3 A comparison of the results of our study with the two other large studies looking at the natural history of conjunctival naevi $[2,3]$.

\begin{tabular}{|c|c|c|c|}
\hline Variable & This study & $\begin{array}{l}\text { Shields } \\
\text { et al. [2] }\end{array}$ & $\begin{array}{l}\text { Levecq } \\
\text { et al. [3] }\end{array}$ \\
\hline $\begin{array}{l}\text { Age at presentation, mean } \\
\text { (range), years }\end{array}$ & $12(4-19)$ & $30(2-93)$ & $31(1-90)$ \\
\hline Male & $47(61 \%)$ & $201(49 \%)$ & $140(55 \%)$ \\
\hline Female & $30(39 \%)$ & $209(51 \%)$ & $115(45 \%)$ \\
\hline \multicolumn{4}{|l|}{ Race } \\
\hline White & $24(31 \%)$ & $365(89 \%)$ & $218(85 \%)$ \\
\hline Black & $10(13 \%)$ & $23(6 \%)$ & $32(13.5 \%)$ \\
\hline Asian & $16(21 \%)$ & $8(2 \%)$ & $3(1 \%)$ \\
\hline Mixed & $1(1 \%)$ & 0 & 0 \\
\hline Unknown & $22(29 \%)$ & 0 & 0 \\
\hline Other & $4(5 \%)$ & $14(3 \%)$ & $2(<1 \%)$ \\
\hline \multicolumn{4}{|l|}{ Naevus location } \\
\hline Bulbar & $74(96 \%)$ & $302(72 \%)$ & $\begin{array}{l}170 \\
(66.6 \%)\end{array}$ \\
\hline Caruncle & $3(4 \%)$ & $61(15 \%)$ & $56(22 \%)$ \\
\hline \multicolumn{4}{|l|}{ Quadrant } \\
\hline Temporal & $41(53 \%)$ & $190(46 \%)$ & $89(36 \%)$ \\
\hline Nasal & $32(42 \%)$ & $184(44 \%)$ & $129(52 \%)$ \\
\hline Superior & $4(5 \%)$ & $23(6 \%)$ & $21(8 \%)$ \\
\hline Inferior & 0 & $21(5 \%)$ & $9(4 \%)$ \\
\hline $\begin{array}{l}\text { Largest basal diameter, } \\
\text { mean (range), mm }\end{array}$ & $4.8(1.0-10)$ & $\begin{array}{l}4.1 \\
(0.2-30.0)\end{array}$ & 4.3 \\
\hline \multicolumn{4}{|l|}{ Colour } \\
\hline Brown & $44(57 \%)$ & $271(65 \%)$ & $131(51 \%)$ \\
\hline Tan & $3(4 \%)$ & $80(19 \%)$ & $71(28 \%)$ \\
\hline Amelanotic & $14(18 \%)$ & $67(16 \%)$ & $53(21 \%)$ \\
\hline Mixed & $16(21 \%)$ & 0 & 0 \\
\hline Cysts present & $63(82 \%)$ & $271(65 \%)$ & $146(57 \%)$ \\
\hline Feeder vessels present & $21(27 \%)$ & $137(33 \%)$ & $69(27 \%)$ \\
\hline Intrinsic vessels present & $24(31 \%)$ & $160(38 \%)$ & $54(21 \%)$ \\
\hline Hair present & $1(1 \%)$ & $\begin{array}{l}\text { Not } \\
\text { reported }\end{array}$ & $\begin{array}{l}\text { Not } \\
\text { reported }\end{array}$ \\
\hline $\begin{array}{l}\text { Anterior margin } \\
\text { touching limbus }\end{array}$ & $44(57 \%)$ & $202(48 \%)$ & $104(41 \%)$ \\
\hline $\begin{array}{l}\text { Growth during } \\
\text { observation period }\end{array}$ & $5 / 59(8.5 \%)$ & $\begin{array}{l}10 / \\
149(7 \%)\end{array}$ & $\begin{array}{l}\text { Not } \\
\text { reported }\end{array}$ \\
\hline $\begin{array}{l}\text { Pigment change during } \\
\text { observation period }\end{array}$ & $8 / 59(14 \%)$ & $\begin{array}{l}20 / \\
149(13 \%)\end{array}$ & $\begin{array}{l}\text { Not } \\
\text { reported }\end{array}$ \\
\hline \multicolumn{4}{|l|}{ Reason for excisional biopsy } \\
\hline Patient request & $9(75 \%)$ & $16(10 \%)$ & $62(83 \%)$ \\
\hline Rule out malignancy & $3(25 \%)$ & $144(90 \%)$ & $13(17 \%)$ \\
\hline \multicolumn{4}{|l|}{ Histologic type } \\
\hline Compound nevus & $8 / 12(66.6 \%)$ & $\begin{array}{l}103 / \\
151(68 \%)\end{array}$ & $\begin{array}{l}55 / \\
75(74 \%)\end{array}$ \\
\hline Junctional nevus & $1 / 12(8.3 \%)$ & $5 / 151(3 \%)$ & $4 / 75(5 \%)$ \\
\hline Combined nevus & $1 / 12(8.3 \%)$ & $6 / 151(4 \%)$ & 0 \\
\hline Intraepithelial naevus & $1 / 12(8.3 \%)$ & & 0 \\
\hline
\end{tabular}

Table 3 (continued)

\begin{tabular}{llll}
\hline Variable & This study & $\begin{array}{l}\text { Shields } \\
\text { et al. [2] }\end{array}$ & $\begin{array}{l}\text { Levecq } \\
\text { et al. [3] }\end{array}$ \\
\hline Other naevus & 0 & $34 /$ & $16(21 \%)$ \\
Melanoma & $1 / 12(8.3 \%)$ & $3 / 151(2 \%)$ & 0 \\
\hline
\end{tabular}

Growth of conjunctival naevi was uncommon in this study $(8.5 \%)$. We found a similar incidence of growth to that reported by Shields et al. ( 8.5 versus $7 \%$ ), whose study included paediatric cases; however, only one-third of our patients were Caucasian compared to almost $90 \%$ of those seen at the Wills Eye Hospital, suggesting that ethnicity of the patient is not related to growth of conjunctival naevi. It is important to exclude complexion-related melanosis when diagnosing conjunctival naevi. In only one child did growth of the naevus prompt excisional biopsy; histopathology showed the lesion to be a benign compound naevus. Naevus growth and change in pigmentation in childhood and adolescence have been recognised previously and, alone, are not considered to be signs of malignancy $[2,3]$. This is the reason why only two out of 13 cases in our series that demonstrated a change in colour or size underwent surgical removal. Depigmentation is not a worrisome feature in conjunctival naevi, as reflected by the fact that all naevi that depigmented in this study showed cysts. It is possible that a change in the size or number of cysts leads the clinician to suspect that these naevi are depigmenting. It has been suggested that changes in the colour or size of conjunctival naevi might be due to inflammation in the naevus rather than malignant change. Zamir et al. [7] found that $75 \%$ of conjunctival naevi in children have some degree of inflammation and this tends to occur mostly in children with a history of allergic/vernal conjunctivitis.

Limitations of this study include the fact, although all children had photographs taken at multiple visits, some children did not have photographs taken at every visit, though clinical examination always compared against the previously taken photographs. We therefore had to rely on clinical notes documenting lack of growth in some cases where photographs were not available. Another limitation is the follow-up. At the time the patients in this study were seen, anterior segment OCT was not performed routinely in our practice. In future, it would be helpful to repeat this study using anterior segment OCT, which is being used more widely [8]. This imaging is likely to increase sensitivity with which changes in size, particularly thickness, are detected and is more reflective of how we are likely to be practicing ocular oncology in the future.

This study provides reassurance that clinical features may allow conjunctival naevi to be distinguished from 
melanomas in children and adolescents, as is the case in adults. In addition, some benign lesions show growth, which in children is not necessarily an indication of malignant transformation. At this time, during the COVID19 pandemic, every attempt should be made to reduce the number of face-to-face consultations at tertiary referral centres. This study will hopefully give reassurance to clinicians that children with conjunctival naevi showing benign clinical features can safely be monitored in the community. Surgical removal is rarely required unless suspicious clinical features are present.

\section{Summary}

\section{What was known before}

- The natural history of conjunctival naevi in adults has been characterised before.

- Due to limited knowledge about the natural history of conjunctival naevi in children these are often followed up for long periods of time in tertiary referral centres.

\section{What this study adds}

- We characterise the natural history of conjunctival naevi in children and adolescents.

- We provide reassurance that prolonged follow-up may not be necessary.

Funding The research was supported by the National Institute for Health Research (NIHR) Biomedical Research Centre based at
Moorfields Eye Hospital NHS Foundation Trust and UCL Institute of Ophthalmology. The views expressed are those of the author(s) and not necessarily those of the NHS, the NIHR or the Department of Health.

\section{Compliance with ethical standards}

Conflict of interest The authors declare that they have no conflict of interest.

Publisher's note Springer Nature remains neutral with regard to jurisdictional claims in published maps and institutional affiliations.

\section{References}

1. Shields CL, Sioufi K, Alset AE. Clinical features differentiating benign from malignant conjunctival tumors in children. JAMA Ophthalmol. 2017;135:215-24.

2. Shields CL, Fasiuddin AF, Mashayekhi A, Shields JA. Conjunctival nevi: clinical features and natural course in 410 consecutive patients. Arch Ophthalmol. 2004;122:167-75.

3. Levecq L, De Potter P, Jamart J. Conjunctival nevi clinical features and therapeutic outcomes. Ophthalmology. 2010;117:35-40.

4. Shields CL, Shields JA, Gündüz K, Cater J, Mercado GV, Gross N, et al. Conjunctival melanoma: risk factors for recurrence, exenteration, metastasis and death in 150 consecutive patients. Arch Ophthalmol. 2000;118:1497-507.

5. McDonnell JM, Carpenter JD, Jacobs P, Wan WL, Gilmore JE. Conjunctival melanocytic lesions in children. Ophthalmology. 1989;96:986-93.

6. Gerner N, Nørregaard JC, Jensen OA, Prause JU. Conjunctival naevi in Denmark 1960-1980. A 21-year follow-up study. Acta Ophthalmol Scand. 1996;74:334-7.

7. Zamir E, Mechoulam H, Micera A, Levi-Schaffer F, Pe'er J. Inflamed juvenile con-junctival nevus: clinicopathological characterization. Br J Ophthalmol. 2002;86:28-30.

8. Shields CL, Belinsky I, Romanelli-Gobbi M, Guzman J, Mazzuca Jr D, Green WR, et al. Anterior segment optical coherence tomography of conjunctival nevus. Ophthalmology. 2011;118:915-9. 